[*] Prof. Dr.-Ing. Lothar Wondraczek

Otto-Schott-Institute

University of Jena, Fraunhoferstrasse 6, Jena 07743, Germany

E-mail: lothar.wondraczek@uni-jena.de

Phone: $+49(0) 3641948500$

\title{
Local deformation of glasses is mediated by rigidity fluctuation and granularity
}

Omar Benzine ${ }^{1}$, Sebastian Bruns ${ }^{2}$, Zhiwen Pan $^{1}$, Karsten Durst ${ }^{2}$, Lothar Wondraczek ${ }^{1,3 *}$

${ }^{1}$ Otto Schott Institute of Materials Research, University of Jena, Fraunhoferstrasse 6, 07743 Jena, Germany

2 Department of Materials Science, Physical Metallurgy, Technical University of Darmstadt, Alarich-Weiss-Straße 2, 64287 Darmstadt, Germany

3 Abbe Center of Photonics, University of Jena, Albert-Einstein-Strasse 6, 07745 Jena, Germany

\section{Abstract}

Microscopic deformation processes lie at the origin of defect formation on glass surfaces, thus determining the material's resistance to scratching and mechanical failure. While the macroscopic strength of most glasses is not directly depending on material composition, local deformation and flaw initiation are strongly affected by chemistry and atomic arrangement. Aside empirical insight, however, the structural origin of the fundamental deformation modes remains largely unknown. Experimental methods which probe parameters on short or intermediate length-scale such as atom-atom or super-structural correlations are typically applied in the absence of alternatives. Drawing on recent experimental advances, we now probe spatial variations in the low-frequency vibrational density of states which result from sharp contact deformation of vitreous silica. From direct observation of deformation-induced variations on the characteristic length-scale of molecular heterogeneity, we argue that rigidity fluctuation on the scale of a few nanometers governs the deformation process of inorganic glasses. 


\section{Introduction}

The mechanical behaviour of glass surfaces has become a subject of large and widespread interest [1]. Most prominently, this has been triggered by rapidly emerging products which rely on haptic interaction and, hence, are prone to surface damage, for example, touch panels, personal electronic devices, and thin and flexible displays. Overcoming the simplistic notion of ultimate brittleness, it has now been understood that the resistance to early-stage surface defects can be tailored through chemical composition, and glasses with improved damage resistance have been identified. However, lacking mechanistic knowledge on local deformation processes still limits conceptual tools for material design.

Since the first report on plastic deformation of optical glasses [2], the seminal subsequent differentiation into "normal" and "anomalous" glasses [3] and early intentional developments of silicate glasses with reduced brittleness [4], a large number of studies has been relying on micro- and nano-indentation to elucidate the interplay between glass chemistry and the material's response in sharp-contact situations. Focusing on vitreous silica as an archetypal model, Perriot et al. [5] were the first to carry-out Raman spectroscopic mapping of Vickers indentation residual imprints. Drawing on studies of Suguira et al. [6] which revealed an analytical relationship between the shift of the characteristic D2 Raman band and the degree of structural densification, they found a heterogeneous distribution of densification around the residual imprint. Similar work was subsequently conducted on other commercially relevant glasses [7,8], and extended by Tran et al. [9] through 3D micro-Brillouin mapping.

These experimental mapping data have led to the development of constitutive descriptions of the indentation process $[10,11,12,13]$ and associated cracking phenomena [14]. However, a view at the underlying structural reactions was obtained only on the short-range level, for example, demonstrating variations in the first coordination shell of network-forming ion species. Although it has been shown that these parameters are insufficient to explain macroscopic densification and shear 
$[15,16]$, in the absence of further insight, short-range parameters have commonly been taken as indicators for the structural origin of mechanical behaviour [1].

In order to bridge the disagreement between structural observations on short range and macroscopic behaviour, we now assess the material's response to mechanical contact on intermediate (nanometer) length-scale, considering deformation-induced variations in the low-frequency vibrational anomaly of glasses.

As an apparently universal feature, glassy materials exhibit an excess in the vibrational density of states (VDoS) as compared to the Debye model [17] of crystalline materials. This excess manifests in the form of a broad band in the reduced vibrational density of states $\mathrm{g}(\omega) / \omega^{2}$, known as Boson peak and observable in the frequency regime of $\sim 10-100 \mathrm{~cm}^{-1}(0.1-3 \mathrm{THz})$, for example, by Raman spectroscopy [18] or low-temperature calorimetry [19]. Recent studies (still focusing mostly on vitreous silica) have been extending the experimental perspective using hyper-Raman spectroscopy [20,21], far-infrared spectroscopy [22], terahertz timedomain spectroscopy [23], or inelastic scattering of X-rays [24] and neutrons [25].

The origin of the Boson peak remains debated, mostly between three general aspects [26, 27, 28]: spatially fluctuating elastic constants $[27,29,30]$, dynamic heterogeneity and the glass transition [31], and coupling of sound waves to quasilocalized modes of defect states $[32,33]$. In all three considerations, the Boson peak is directly or indirectly related to structural heterogeneity of the solid glass, typically on the scale of a few nanometers. It has further been shown extensively that its frequency $\left(\omega_{B P}\right)$ and intensity $\left(I_{B P}\right)$ depend not only on glass composition [34], but also on thermal history $[35,36]$, pressure $[37,38,39,40,41,42,43]$ and the presence of different polymorphic states [44,45]. Regarding the pressure-dependence, analyses have been carried-out primarily in quasi-isostatic conditions, in situ using diamond anvil cells or ex situ through high temperature/high pressure multi-anvil setups $[27,39]$.

In analogy to lateral or 3D mapping of short-range order during indentation deformation, and using isostatic compaction data for reference, we now map variations on the Boson peak as they result from local structural deformation. Drawing on recent progress in low-frequency Raman spectroscopy for facile data 
acquisition and a new constitutive model for glass densification, we reveal the role of structural heterogeneity in the deformation process of silicate glasses.

\section{Results}

\section{Raman scattering and local densification}

Normalized Raman spectra as obtained from the top face of a silica glass sample after Vickers indentation are shown in Fig. 1a. All spectra exhibit the characteristic band shape of vitreous silica. It comprises of three regions: (1) the high-frequency regime between $850 \mathrm{~cm}^{-1}$ and $1400 \mathrm{~cm}^{-1}$, dominated by stretching vibrations of $\mathrm{SiO}_{2}$ sub-units at $\sim 800 \mathrm{~cm}^{-1}, 1080 \mathrm{~cm}^{-1}$ and $1200 \mathrm{~cm}^{-1}$ [46], (2) the region located between $200 \mathrm{~cm}^{-1}$ to about $850 \mathrm{~cm}^{-1}$ with the main band $(\mathrm{MB})$ at $\sim 435 \mathrm{~cm}^{-1}$ (symmetric rocking of bridging oxygen species) [47] and the two defect bands D1 and D2 at $\sim 490$ and $601 \mathrm{~cm}^{-1}$ (symmetric breathing modes of four- and three-membered $\mathrm{SiO}_{4}$ rings, respectively) [48], and (3) the low-frequency region of $10-200 \mathrm{~cm}^{-1}$ with the pronounced Boson peak (BP) located at $50-80 \mathrm{~cm}^{-1}$.

a.

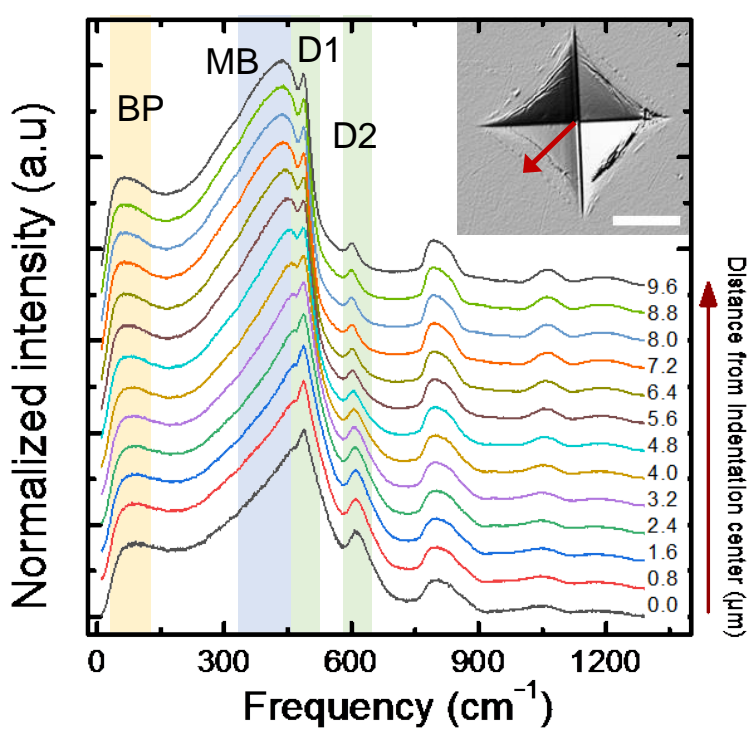

b.

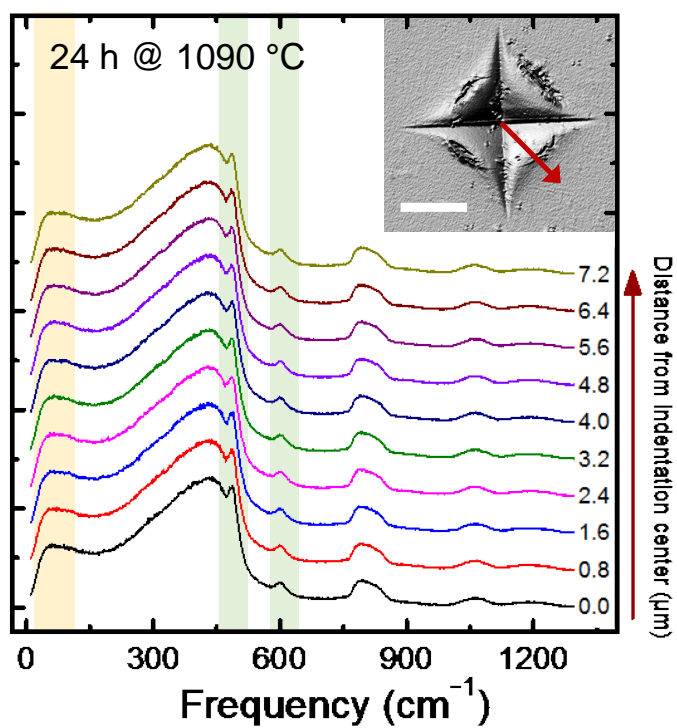

Fig. 1. Raman scattering of vitreous silica after indentation deformation. Raman spectra were collected by scanning along the face of a Vickers indent (created by loading with $2.94 \mathrm{~N}$ for $15 \mathrm{~s}$ ) as indicated in the insets, before (a) and after annealing at $\sim 0.9 T_{\mathrm{g}}$ for $24 \mathrm{~h}$. Curves show the Stokesside of normalized Raman spectra recorded at a step-width of $0.4 \mu \mathrm{m}$. For better visibility, we display only every second curve (0.8 $\mu \mathrm{m}$ spacing). The locations of the Boson peak (BP), the silica main band $(\mathrm{MB})$ and the defect bands D1 and D2 are highlighted. Scale bars in the insets are $10 \mu \mathrm{m}$. 
The Boson peak represents the collective motion of a great number of atoms [49]. Indentation deformation of glass surfaces is generally taken as a result of two processes: densification on molecular or intermediate length-scale, and shear flow on longer scale [50]. The atomic packing density and Poisson's ratio indicate which of the two reactions is dominating [87]. Here, vitreous silica with its low Poisson ratio belongs to the so-called anomalous glasses in which a very high degree of densification is possible, i.e., up to $85-92 \%$ [51]. In order to judge the individual contributions of densification and shear to the deformation-induced variation of Boson Peak frequency, low-frequency Raman mappings are compared before (Fig. 1a) and after annealing (Fig.1b, annealed for $24 \mathrm{~h}$ at $0.9 \times T_{\mathrm{g}}$ ). This assumes that structural compaction fully relaxes during annealing, whereas shear deformation does not.

While the principal Raman signature remains unchanged before and after annealing, during heat treatment, stress relaxation is accompanied by structural relaxation [51] and the indentation-induced structural change recovers to a large extent. This confirms previous observations that indentation deformation occurs primarily through structural compaction.

In order to correct first-order Raman scattering data for the influences of temperature and frequency, the measured intensity $I_{m e s}(\omega, T)$ is rewritten in reduced form as the product of the reduced density of states $g(\omega) / \omega^{2}$ and the coupling coefficient $C(\omega)$ [52],

$\mathrm{I}^{\mathrm{red}}(\omega)=\frac{\mathrm{I}_{\text {mes }}(\omega, \mathrm{T})}{[\mathrm{n}(\omega, \mathrm{T})+1] \omega}=\mathrm{C}(\omega) \frac{\mathrm{g}(\omega)}{\omega^{2}}$

In Eq. 1, $n(\omega, T)=[\exp (\hbar \omega / k T)-1]^{-1}$ is the Bose-Einstein population factor for frequency $\omega$ and temperature $T, \hbar$ and $k$ are the reduced Planck and Boltzmann constants, respectively. While for pristine vitreous silica, a nearly linear frequencydependence is usually observed for $C(\omega)$ over the frequency range of $0.5 \omega_{B P}$ to $1.5 \omega_{B P}[34,53,54]$, Carini et al. [27] suggested a dependency of the form $C(\omega) \propto \omega^{\alpha}$ (within 10-120 $\mathrm{cm}^{-1}$, avoiding quasi-elastic scattering at $<10 \mathrm{~cm}^{-1}$ and optical modes at $>120 \mathrm{~cm}^{-1}$ ) for densified silica. The exponent $\alpha$ was found to vary with the degree of densification $\left(\rho / \rho_{0}\right)$ and was fit as $\left.\alpha=\mathrm{A}+\left[\mathrm{B} \times\left(\rho / \rho_{0}\right)^{C}\right)\right]$ with constants $\mathrm{A}, \mathrm{B}$ and C. In order to derive $g(\omega) / \omega^{2}$ from Eq (1), we now first estimate the degree of 
densification corresponding to each Raman spectrum. For this, we use an empirical relation which relates the $\mathrm{D} 2$ band position to the densification ratio $[5,6]$,

$$
0.143 \log \left(\frac{\rho}{\rho_{0}}\right)=\log \left(\frac{\omega}{\omega_{0}}\right)
$$

Here, $\omega$ and $\omega_{0}$ are the observed and the reference (pristine) frequency locations of the D2 band. The evaluation through Eq. 2 does not differentiate between plastic and elastic contributions [5]. However, it has been shown that the D2 band frequency is only marginally affected by residual elastic strain [55]. In Fig. 2, the thus-obtained densification data are compared to FEM modelling results.

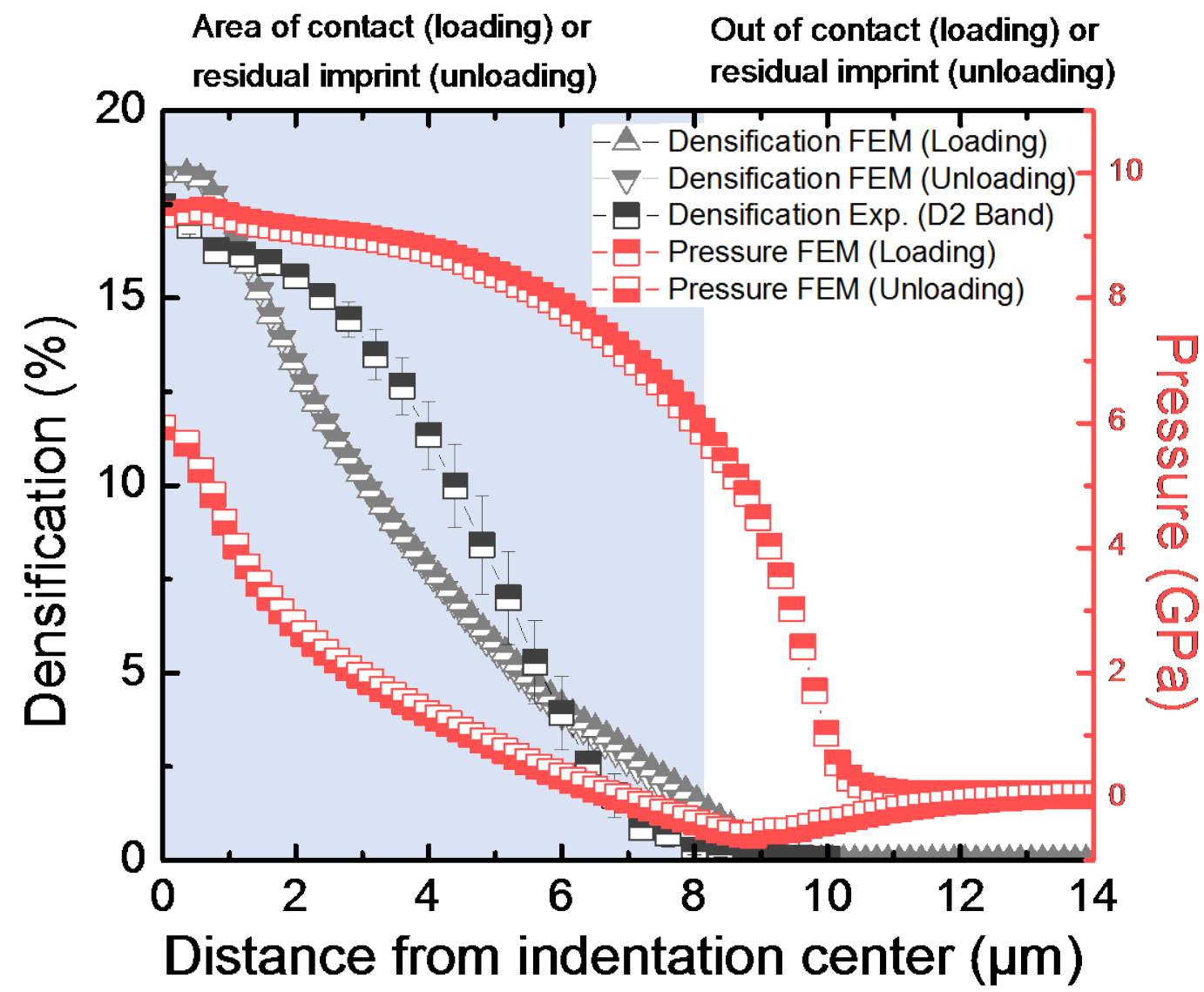

Fig. 2. Indentation-induced densification. Densification (PEQC4) and pressure distribution as function of the distance from the indentation center. Experimental data are according to Eq. 2. FEM simulation results are provided during holding the peak load and after unloading for a Vickersequivalent conical indenter with an opening angle of $70.29^{\circ}$. The shaded area marks the contact/imprint region. 
Finite element modelling of the indentation process reveals an inhomogeneous stress distribution beneath the indenter, both during and after loading. The equivalent pressure is largest at the indenter tip and decreasing towards the edges. In the vicinity of the contact region a tensile stress component in radial direction is arising, counteracting the compressive stress distribution beneath the indenter tip. This stress component causes a minimum in the equivalent pressure at a distance of roughly $9 \mu \mathrm{m}$. Densification is at its maximum of $\sim 18.3 \%$ (FEM) or $17.4 \%$ (Raman D2 experiment) right in the centre of the indent. Also the lateral expansion of the compaction field matches the Raman measurements very well. In the intermediate section, densification is slightly underestimated by FEM, which might be due to the many simplifications in the model. For example, transient variations in the elastic properties are still neglected. We further notice that the maximum densification observed here is somewhat smaller than the expected saturation value of about $21 \%$ which has been reported for vitreous silica [87]. This is similar to other observations $[5,10]$ and may be attributed to shear flow yielding which limits the maximum hydrostatic component beneath the indenter.

\section{Vibrational density of states and Boson peak}

From densification data, the exponent $\alpha$ and subsequently $C(\omega)$ were derived for calculating the reduced vibrational density of states, $g(\omega) / \omega^{2}$ (Fig. 3). 


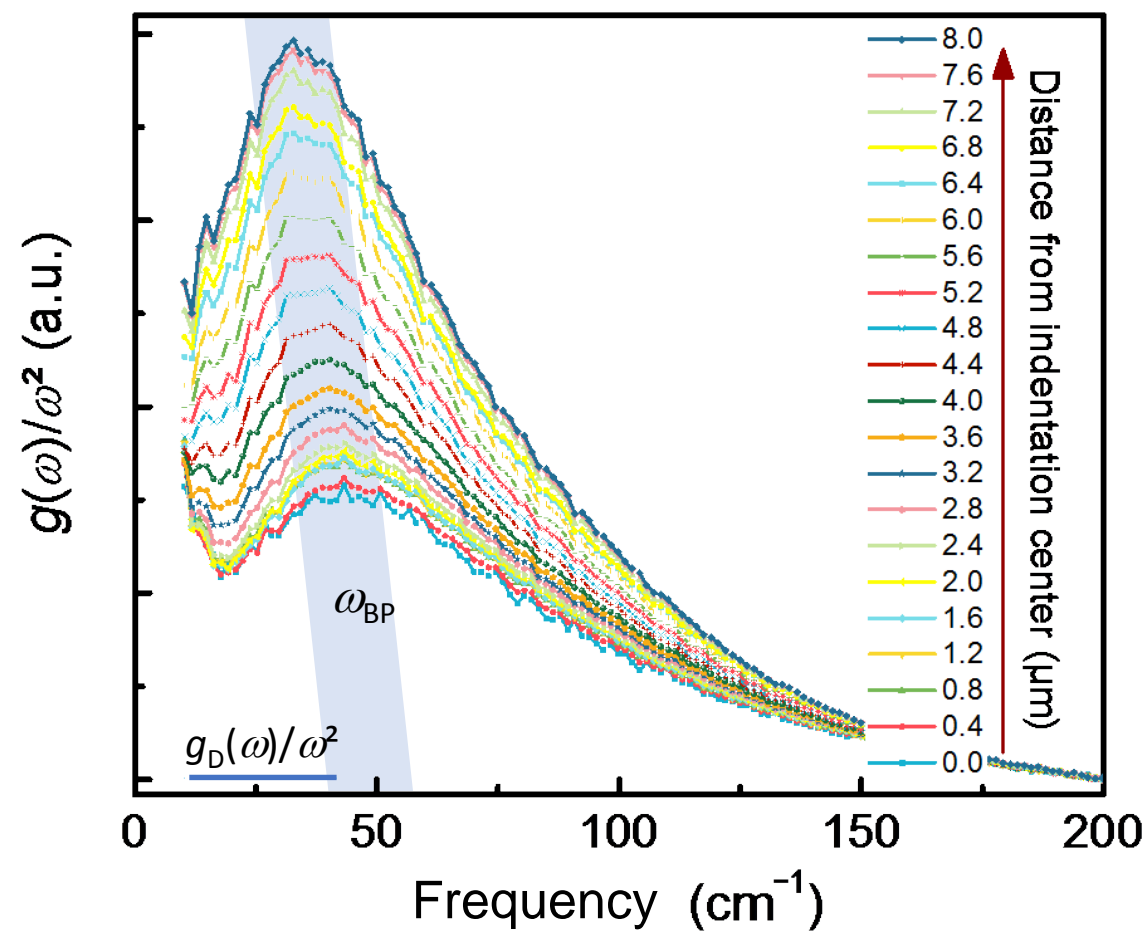

Fig. 3. Reduced vibrational density of states $g(\omega) / \omega^{2}$ of silica after indentation deformation. Data collected by low-frequency Raman spectroscopy at varying distances from the centre of residual imprint (see also Fig. 1). The Debye level $g_{\mathrm{D}} / \omega^{2}$ of the centre spectrum is shown for reference.

All spectra exhibit the excess in $\mathrm{g}(\omega)$ as compared to the Debye prediction which is typical for glassy materials (with $g_{\mathrm{D}}(\omega)=3 \omega^{2} / \omega_{D}^{3} \approx \omega^{2}$, with the Debye frequency $\left.\omega_{D}\right)$. In order to estimate the Boson peak frequency $\omega_{B P}$ quantitatively the reduced intensity was fitted with a log-normal function [34, 56], taking into account the asymmetric shape of the Boson peak. In Fig. 3, $\omega_{\mathrm{BP}}$ progressively shifts to lower frequency with increasing distance from the centre of the indent, i.e., from $\sim 45 \mathrm{~cm}^{-1}$ within the region of highest densification to about $33.7 \mathrm{~cm}^{-1}$ in a radial distance of $8 \mu \mathrm{m}$ from the centre of the indent. The latter value corresponds to the value of the Boson peak frequency $\omega_{B P}$ in typically found in pristine silica $\left(33.5 \mathrm{~cm}^{-1},[21,27,54]\right)$. The shift of $\omega_{\mathrm{BP}}$ is accompanied by a significant increase of Boson peak intensity (eVDOS), signifying a reduction of low-energy vibrational modes with increasing stress. Interestingly, the change in Boson peak intensity (e-VDOS) is significantly more pronounced than the intensity variations seen on the main vibrational bands (Fig. 1). This observation suggests that the low-energy vibrational modes (Boson peak) are 
affected more strongly through local deformation than the higher energy vibrational modes and, thus, the short-range structural arrangement.

\section{Indentation-induced structural compaction}

The heterogeneous stress distribution shown in Fig. 2 leads to inhomogeneous distribution of densification around the residual imprint. During high-pressure loading of 5- $10 \mathrm{GPa}$, the packing density of the $\mathrm{SiO}_{2}$ network irreversibly increases $[57,58,59]$. It has been suggested that this is driven by topological reconstruction at intermediate length-scale, in particular, relating to the distribution of rings of cornersharing $\mathrm{SiO}_{4}$ tetrahedra with different size (the proof of the existence of those rings is based mainly on Raman scattering observations of the D1 and D2 bands, Fig. 1). The inter-tetrahedron angle $\theta$ usually takes a wide range of values from within $120^{\circ}$ to $180^{\circ}$ with a most frequent state of $\theta \sim 144^{\circ}$ [60]. This wide angle distribution results also in a wide distribution of possible ring configurations, i.e., from 3-fold to 10 -fold $[61,62,63]$, with a maximum around 6 -fold. For permanently densified silica, neutron diffraction has shown that the $\mathrm{SiO}_{4}$ tetrahedron itself is only marginally affected, at least within the present range of stress [64]. Instead, the inter-tetrahedral angle and, thus, the Si-Si distance decrease significantly with proceeding compaction, for example, from $\sim 144^{\circ}$ to about $\sim 138-139^{\circ}$ at $\sim 21 \%$ densification $[58,59]$.

Considering the present observation of a reduction of excess low-energy vibrational modes (Fig. 3), it was suggested from INS and hyper-Raman experiments that lowfrequency vibrations might correspond to the rotational motions of clusters of several tetrahedra [21,65,66]. According to Hehlen et al. [21], however, these modes are not active in infrared and Raman spectroscopy. The low-frequency Raman signal can be interpreted in the frame of the soft potential model (SPM) which is assuming that the low-frequency dynamics of glasses are characterized by the presence of additional quasi-localized vibrations (QLV) [42]. Raman-active QLVs could result from to the $\mathrm{SiO}_{4}$ librational motions coupled to the acoustic continuum [67]. The main effect of densification is therefore to compact the network of tetrahedra by reducing the free volume that exist in the silica network, but without affecting the elementary structural units. The decrease in the size of the rings and the reduction in dynamic degrees of freedom impede the rotational motions of tetrahedra linked in the rings, which are 
taken as the main source of the Boson peak. The increase of the stiffness leads to the suppression of soft vibrations and to an increase of their frequency $\omega_{\mathrm{BP}}$ as observed in Fig. 3.

In a further consideration $[26,29,30]$, thus generated random spatial fluctuations of the transverse elastic constants induce excess vibrational states at low frequency. Then, the acting pressure reduces the amplitude of fluctuation, leading to the observed shift of the Boson peak and to a decrease of its intensity relative to the Debye level. As discussed by Flores-Ruiz and Naumis [68], the frequency of the Boson peak shifts to higher frequency and, at the same time, its intensity decreases with increasing network rigidity. Also this is in line with the present observation of the effect of indentation-induced compaction (Fig. 3).

\section{Breakdown of CMT predictions and role of rigidity fluctuation}

As noticed in Fig. 2, indentation-induced stress affects material density. Density variations, on the other side, affect other properties of the material, including the elastic constants [69]. Therefore, comparison between experimental data and the predictions of theoretical models requires to consider the changes also in these parameters. Here, we now compare the Boson peak frequency $\omega_{B P}$ and intensity (eVDOS) with predictions of the continuous medium transformation (CMT) model. First, in order to check whether the shift of the Boson Peak frequency $\omega_{\mathrm{BP}}$ (Fig. 3) can be ascribed completely to changes in elastic medium transformations as predicted by the CMT model, we compare the dependence of Boson peak frequency $\omega_{\mathrm{BP}}$,Debye frequency $\omega_{\mathrm{D}}$ (from Eq. 3) and the elastic constants $\left(V_{\mathrm{T}}\right.$ and $\left.V_{\mathrm{L}}\right)$ as functions of the density around the residual imprint. Results of this comparison are plotted in Fig. $\mathbf{4 a}$.

$$
\omega_{D}=\left(6 \pi^{2} \rho N_{A} m / M\right)^{1 / 3} V_{D}
$$

, with the Avogadro number $N_{A}$, the average number of atoms per molecule in the sample $m$, the average molar weight $M$, and the density $\rho$ as obtained using the shift of the D2 band. $V_{D}$ is the Debye sound velocity obtained by averaging $V_{L}$ and $V_{T}$ through $\left(3 / V_{D}{ }^{3}\right)=\left(1 / V_{L}^{3}+2 / V_{T}^{3}\right)$. Sound velocity data are taken from Ref. [70].

The variation of the Boson peak intensity under mechanical stress is usually compared to the variation of Debye level $\omega_{\mathrm{D}}^{-3}$ (with $\mathrm{g}_{\mathrm{D}}(\omega) / \omega^{2} \approx \omega_{\mathrm{D}}^{-3}$ ) [71,72], 
although simulation studies have shown the limits of this approach [73]. In the alternative CMT model, the comparison is done between the observed $g(\omega)$ and a modelled $g(\omega)^{\mathrm{CMT}}[74]$,

$$
\mathrm{g}(\omega)^{\mathrm{CMT}} / \omega^{2}=\left(1 /\left(\mathrm{d} \times \mathrm{V}_{\mathrm{D}}^{3}\right)\right)=(3 \mathrm{~d})^{-1}\left(\frac{1}{\mathrm{~V}_{\mathrm{L}}^{3}}+\frac{2}{\mathrm{~V}_{\mathrm{T}}^{3}}\right)
$$

Results are plotted in Fig. 4b.
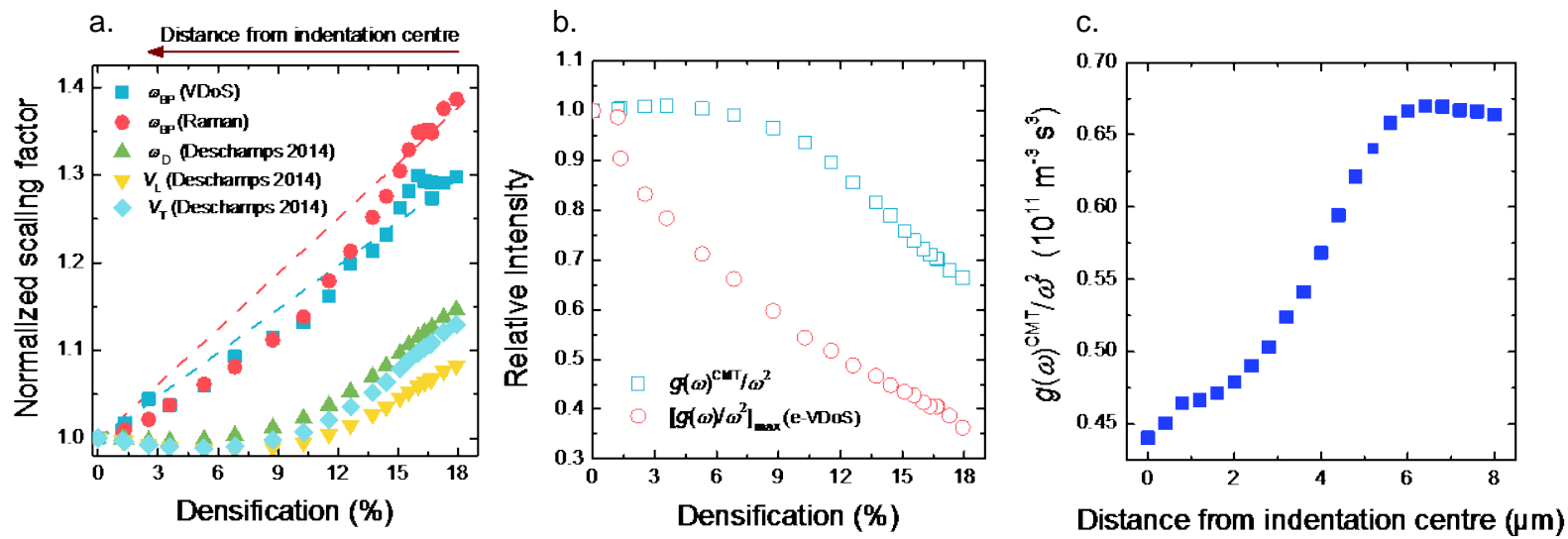

Fig. 4. Data evaluation according to the continuous medium transformation (CMT) model. (a) Variation of the scaling factors relative to Boson peak frequency $\omega_{\mathrm{BP}}$ (VDoS), $\omega_{\mathrm{BP}}$ (Raman), Debye frequency $\omega_{D}$ and elastic constants $\left(V_{T}\right.$ and $\left.V_{L}\right)$ as a function of densification. Dashed lines in (a) are drawn to guide the eye. (b) Variation of the relative intensity of $g(\omega) / \omega^{2}$ (e-VDoS) and $g(\omega)^{\mathrm{CMT}} / \omega^{2}$ as functions of densification. (c) Variation of $\mathrm{g}(\omega)^{\mathrm{CMT}} / \omega^{2}$ as a function of the distance from the centre of the indenter. In (a) and (b), all data were normalized to their normal density value (corresponding to non-densified silica).

Fig. $4 a$ reveals that the density dependence of scaling factors for Boson peak frequency $\omega_{\mathrm{BP}}$ (VDoS), $\omega_{\mathrm{BP}}$ (Raman), the Debye frequency $\omega_{\mathrm{D}}$ and the elastic constants $V_{\mathrm{T}}$ and $V_{\mathrm{L}}$ is approximately linear. Variations on the Boson peak frequency $\omega_{\mathrm{BP}}$ (VDoS, Raman) as they are induced by indentation show a stronger density dependence than the Debye frequency or the sound velocities. A similar trend was previously seen in isostatically compressed silica [39, 67]. In Fig. 4b, the dependence of the Boson peak intensity and the predicted intensity according to CMT on densification are shown. We notice that (e-VDoS) decreases very quickly with densification, while CMT predicts a more complex trend, similar to earlier observations $[74,75]$. For the present case, both Fig. 4a-b therefore show clearly that the Boson peak frequency shift and intensity variations induced by indentation cannot 
be attributed alone to modifications of a continuous elastic medium. Instead, they are related to local structural transformations. In this context, Mantisi et al. [72] suggested that the deviation from the prediction of the CMT model is a result of two contributions (Eq. 5): changes in elastic properties and in additional vibrations due to structural homogenization which manifest in a reduction of the characteristic lengthscale of heterogeneity $\xi$,

$$
\frac{\Delta \omega_{B P}}{\omega_{B P}}=\frac{\Delta V_{T}}{V_{T}}+\frac{\Delta \xi}{\xi}
$$

The model of a non-continuous structure (underlying Eq. 5 [76]) ascribes the additional vibrations which are at the origin of the Boson peak to material heterogeneity on the nanometric scale. This draws an analogy between the lowfrequency Raman scattering spectra of glasses and heterogeneous materials containing nanoparticles [77]. The system is assumed to consist of cohesive domains in which the atoms are strongly linked to each other, and by softer inter-domain regions. The Boson peak arises from hybridization of acoustic modes with quasilocalized modes around those elastic heterogeneities. The size of the elastic heterogeneities is very similar to the length-scale of dynamic heterogeneity in the liquid state [78]. In this context, the decrease of the VDoS in the most compacted areas during indentation deformation (Fig. 3) is due to weaker hybridization. This results from a decrease of the elastic contrast between the rigid domains and the soft domains. The characteristic length scale $\xi$ is now determined from the ratio of the transverse sound velocity $V_{\mathrm{T}}$ and $\omega_{B P}$ (Fig. 5). Noteworthy, this evaluation does not take into account geometric aspects. A geometry factor $S$ is sometimes included so that $\xi_{\text {corr }}=S\left(V_{T} / \omega_{B P}\right)$, with $S=0.8$ for spherical domains or $S=0.5$ for linear domains [76]. Without knowing the actual shape, a mean value $S=0.65$ is usually taken. 


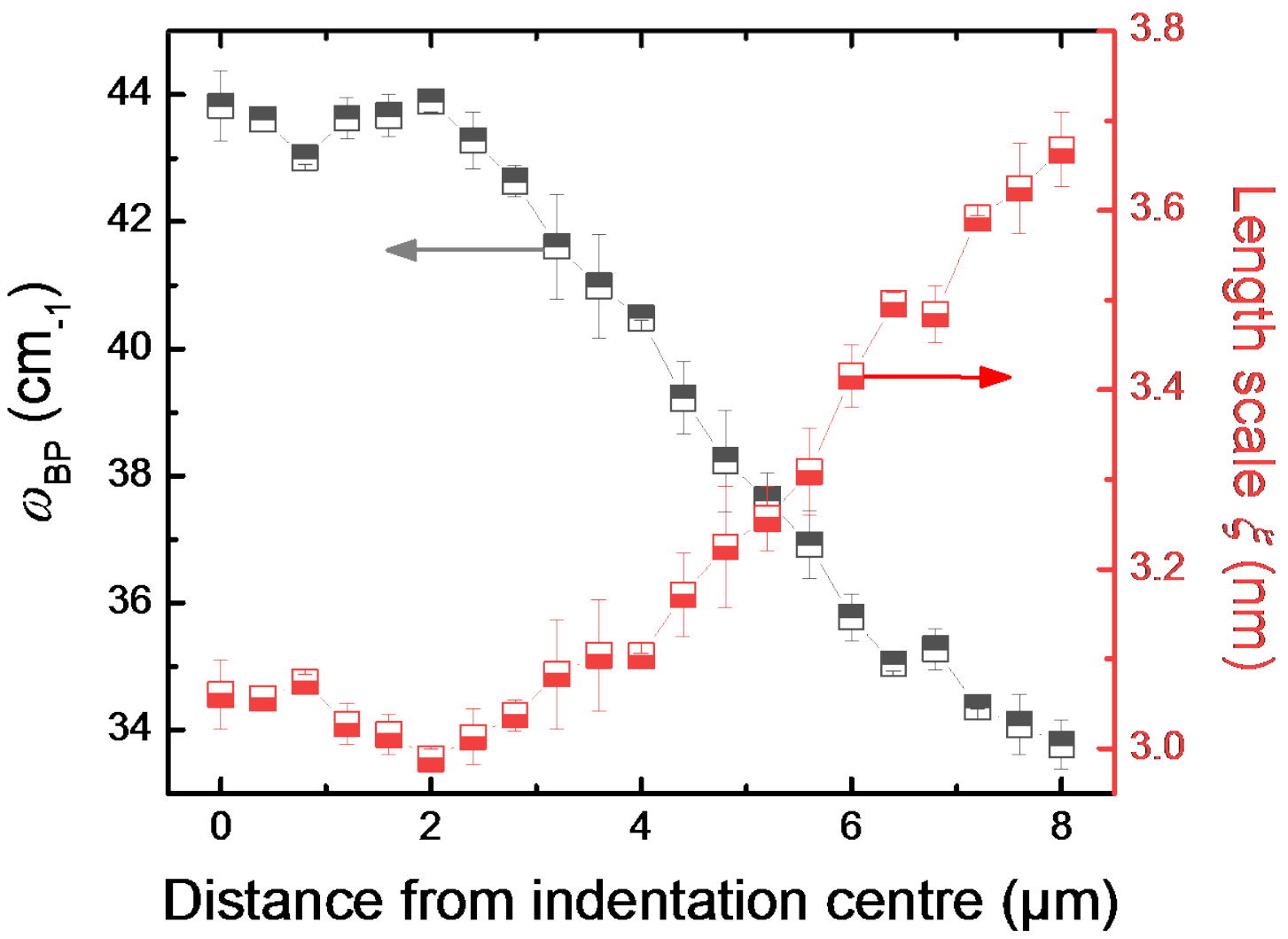

Fig. 5. Effect of indentation-deformation on local structural heterogeneity. Variation of Boson peak frequency $\omega_{B P}$ and the associated length scale $\xi$ with the distance from the indentation centre.

We notice a clear decrease of $\xi$ from $\sim 3.6 \mathrm{~nm}\left(\xi_{\text {corr }}=2.4 \mathrm{~nm}\right)$ to about $3.1 \mathrm{~nm}\left(\xi_{\text {corr }}=\right.$ $1.98 \mathrm{~nm}$ ) between edge and centre of the indentation imprint. This corresponds to a reduction of about $18 \%$. The length scale value in the non-densified area corresponds to about 20 inter-atomic spacings [79] and is in good agreement with simulation data $(\xi=3.3 \mathrm{~nm}[80])$. Furthermore, we see a saturation in the highpressure region of up to $\sim 4 \mu \mathrm{m}$ from the indentation centre, marking the upper limit of the homogenization process. Also the latter observation is in agreement experimental studies on isostatically compacted silica [7]. It is widely assumed that $\xi$ is directly related to a structural correlation length [81, 82]. An empirical proportionality was suggested between $\xi$ and the inverse width of the first sharp diffraction peak (FSDP) [37, 81], whereby the latter is thought to arise from the periodicity of rings of $\mathrm{SiO}_{4}$ tetrahedra [83]. However, the correlation with FSDP annular position seems more complex [84]. 


\section{Discussion}

We considered the structural origin of microscopic deformation processes on glass surfaces in sharp contact situations. Through dissipation of mechanical energy, these processes (in particular, structural compaction and shear) determine the material's resistance to flaw initiation. Acting as local stress amplifiers, such flaws are responsible for mechanical failure and, thus, the macroscopic strength of glass products.

Initially focusing on vitreous silica as a material of technical as well as fundamental importance enabled exclusion of the contributions of chemical fluctuations in the analysis. Spatially-resolved Raman spectroscopy was employed to monitor structural variations induced by micro-indentation. In the low-frequency regime, this provided access to variations in the excess of the vibrational density of states which is inherent to vitreous materials relative of Debye solids. Through scaling of Raman scattering maps, deformation-induced shifts in the length-scale of rigidity fluctuations and fluctuation contrast are revealed in the position and intensity, respectively, of the Boson peak. The extent of these variations corresponds to the degree of local structural densification. This overcomes the limitations of previous observations on short and intermediate length-scale which could not explain the overall extent of deformation. We conclude that inherent fluctuations in rigidity occurring on the scale of a few nanometers control the deformation reactions in vitreous silica and other glassy materials. This observation also holds for the more prolific glass families with widespread technological relevance, in which the magnitude of length-scale variation depends on the individual contributions of densification and shear deformation. The present observations open a new route for tailoring ultra-strong glasses with extreme defect resistance, answering to the major industry challenge towards modern glass applications.

\section{Materials and Methods}

Commercial-grade silica glass $v$ - $\mathrm{SiO}_{2}$ (Suprasil 2, Heraeus) specimens were indented with a Struers Duramin micro-indenter, using a Vickers tip with a load of $2.94 \mathrm{~N}$ and a constant loading time of $15 \mathrm{~s}$. The load was chosen so as to create a large but crack-free permanent imprint (Fig 1) [12]. Structural studies were conducted with a Renishaw Invia micro-Raman spectrometer, equipped with a low-frequency 
notch filter performing down to $\sim 10 \mathrm{~cm}^{-1}$. Samples were excited with a $514 \mathrm{~nm}$ Argon laser at ambient temperature, with a spatial resolution of $<0.5 \mu \mathrm{m}$. The Raman signal was collected with a CCD camera over the frequency range of 10 to $1386 \mathrm{~cm}^{-1}$ with a resolution of $2 \mathrm{~cm}^{-1}$, using a 50x objective. For each spectrum of a $2 \mathrm{D}$ map, an accumulation time of $280 \mathrm{~s}$ was employed.

Finite element modelling (FEM) was performed on ABAQUS [85]. The indentation process was modelled using a two-dimensional axisymmetric model of a Vickersequivalent conical indenter with an opening angle of $70.29^{\circ}$ similar to previous studies by Bruns et al. [14]. All material parameters were assumed to be rateinsensitive and representative of room temperature values. The contact conditions between the rigid Vickers indenter and the sample surface were assumed to be frictionless. Furthermore, elastic isotropy was assumed for fused silica with an elastic modulus of $70 \mathrm{GPa}$ and a Poisson ratio of 0.18 [86]. Anomalous material behaviour was modelled using Drucker-Prager-Cap plasticity with a yield strength under pure shear of $7.5 \mathrm{GPa}$. Details on this model can be found elsewhere [14]. Hereby, densification induced sigmoidal isotropic hardening and saturation of densification as reported by Rouxel et al. [87] were included.

\section{References}

1. L. Wondraczek, J. C. Mauro, J. Eckert, U. Kühn, J. Horbach, J. Deubener, T. Rouxel, Towards ultra-strong glasses. Adv. Mater. 23 (2001) 4578-4586.

2. E. W. Taylor, Plastic deformation of optical glass, Nature 163 (1949) 323-323.

3. A. Arora, D.B. Marshall, B.R. Lawn, M.V. Swain, Indentation deformation/fracture of normal and anomalous glasses, J. Non-Cryst. Solids 31 (1979) 415.

4. J. Sehgal, S. Ito, Brittleness of glass, J. Non-Cryst. Solids 253 (1999) 126-132.

5. A. Perriot, D. Vandembroucq, E. Barthel, V. Martinez, L. Grosvalet, C. Martinet, B. Champagnon, Raman Microspectroscopic Characterization of Amorphous Silica Plastic Behavior. J. Am. Ceram. Soc. 89 (2006) 596-601.

6. H. Sugiura, R. Ikeda, K. Kondo, and T. Yamadaya, Densified Silica Glass After Shock Compression. J. Appl. Phys. 81 (1997) 1651-5.

7. T. Deschamps, C. Martinet, J. L. Bruneel, B. Champagnon, Soda-lime silicate glass under hydrostatic pressure and indentation: a micro-Raman study. J. Phys.: Condens. Matter 23 (2011) 035402.

8. A. Winterstein-Beckmann, D. Möncke, D. Palles, E. I. Kamitsos, L. Wondraczek, A Raman-spectroscopic study of indentation-induced structural changes in technical alkaliborosilicate glasses with varying silicate network connectivity. J. of Non-Cryst. Solids $\mathbf{4 0 5}$ (2014) 196-206.

9. H. Tran, S. Clément, R. Vialla, D. Vandembroucq, B. Rufflé, Micro-Brillouin spectroscopy mapping of the residual density field induced by Vickers indentation in a soda-lime silicate glass. Appl. Phys. Lett. 100 (2012) 231901.

10. G. Kermouche, E. Barthel, D. Vandembroucq, P. Dubujet, Mechanical modelling of indentation-induced densification in amorphous silica. Acta Mater. 56 (2008) 3222-3228.

11. M. Jebahi, D. André, F. Dau, J.-L. Charles, I. Iordanoff, Simulation of Vickers indentation of silica glass. J. Non-Cryst. Solids 378 (2013)15-24. 
12. A. Kassir-Bodon, T. Deschamps, C. Martinet, B. Champagnon, J. Teisseire, Guillaume Kermouche Raman Mapping of the Indentation-Induced Densification of a Soda-LimeSilicate Glass, Int. J. Appl. Glass Sci. 3 (2012) 29-35.

13. G. Molnár, P. Ganster, A. Tanguy, E. Barthel, G. Kermouche: Densification dependent yield criteria for sodium silicate glasses - An atomistic simulation approach. Acta Mater. 111 (2016) 129-137.

14. S. Bruns, K. E. Johanns, H. U.R. Rehman, G. M. Pharr, K. Durst, Constitutive modeling of indentation cracking in fused silica. J Am Ceram Soc. 100 (2017) 1928-1940.

15. L. Wondraczek, S. Sen, H. Behrens, R. E. Youngman, Structure-energy map of alkali borosilicate glasses: Effects of pressure and temperature, Phys. Rev. B 76 (2007) 014202.

16. L. Wondraczek, H. Behrens, Molar volume, excess enthalpy and Prigogine-Defay ratio of some silicate glasses with different (p,T)-histories, J. Chem. Phys. 127 (2007) 154503.

17 .P. Debye, Zur Theorie der spezifischen Wärmen, Ann. Phys. 39 (4) (1912) 789.

18. R.S. Krishnan, The scattering of light in fused quartz and its Raman spectrum, Proc. Indian Acad. Sci. A 37 (1953) 377.

19. P. Flubacher, A.J. Leadbetter, J.A. Morrison, B.P. Stoicheff. The low-temperature heat capacity and the Raman and Brillouin spectra of vitreous silica. J. Phys. Chem. Solids 12 , (1959) 53.

20. B. Hehlen, E. Courtens, R. Vacher, A. Yamanaka, M. Kataoka, K. Inoue, Hyper-Raman Scattering Observation of the Boson Peak in Vitreous Silica. Phys. Rev. Lett. 84 (2006) 5355.

21. B. Hehlen and G. Simon, The vibrations of vitreous silica observed in hyper-Raman scattering. J. Raman Spectrosc. 43 (2012) 1941.

22. B. Guillot, Y. Guissani, Boson Peak and High Frequency Modes in Amorphous Silica, Phys. Rev. Lett. 78 (1997) 2401.

23. S. N. Taraskin, S. I. Simdyankin, S. R. Elliott, J. R. Neilson, and T. Lo Universal Features of Terahertz Absorption in Disordered Materials, Phys. Rev. Lett. 97 (2006) 055504.

24. G. Baldi, V. M. Giordano, G. Monaco, and B. Ruta Sound Attenuation at Terahertz Frequencies and the Boson Peak of Vitreous Silica. Phys. Rev. Lett. 104 (2010) 195501.

25. S. Caponi, A. Fontana, F. Rossi, G. Baldi, and E. Fabiani Effect of temperature on the vibrational density of states in vitreous SiO2: A Raman study, Phys. Rev. B 76 (2007) 092201.

26. W. Schirmacher, Some comments on fluctuating-elasticity and local oscillator models for anomalous vibrational excitations in glasses, J. Non-Cryst. Solids 357 (2011) 518-523.

27. G. Carini, Jr., G. Carini, D. Cosio, G. D'Angelo, F. Rossi, Low temperature heat capacity of permanently densified SiO2 glasses, Phil. Mag. 96 (2016) 761-773.

28. T. Deschamps, C. Martinet, D. de Ligny, J. L. Bruneel, B. Champagnon, Correlation between boson peak and anomalous elastic behavior in GeO2 glass: an in-situ Raman scattering study under high-pressure, J. Chem. Phys. 134 (2011) 234503.

29. W. Schirmacher, G. Ruocco, and T. Scopigno, Acoustic Attenuation in Glasses and its Relation with the Boson Peak, Phys. Rev. Lett. 98 (2007) 025501.

30. W. Schirmacher, Thermal conductivity of glassy materials and the "boson peak", Europhys. Lett. 73 (2006) 892. 
31. W. Götze, M.R. Mayr, Evolution of vibrational excitations in glassy systems. Phys. Rev. E $61(2000) 587$.

32. E. Duval, A. Boukenter, T. Achibat, Vibrational dynamics and the structure of glasses. J. Phys.: Cond. Matt. 2 (1990) 10227.

33. B. Schmid, W. Schirmacher, Raman Scattering and the Low-Frequency Vibrational Spectrum of Glasses. Phys. Rev. Lett. 100 (2008) 137402.

34. M. F. Ando, O. Benzine, Z. Pan, J.-L. Garden, K. Wondraczek, S. Grimm, K. Schuster, L. Wondraczek, Boson peak, heterogeneity and intermediate-range order in binary $\mathrm{SiO}_{2-}$ $\mathrm{Al}_{2} \mathrm{O}_{3}$ glasses, Sci. Rep. 8 (2018) 5394.

35. S. Caponi, A. Fontana, F. Rossi, G. Baldi, E. Fabiani, Effect of temperature on the vibrational density of states in vitreous SiO2: A Raman study, Phys. Rev. B 76 (2007) 092201.

36. B. Rufflé, S. Ayrinhac, E. Courtens, R. Vacher, M. Foret, A. Wischnewski, U. Buchenau, Scaling the Temperature-dependent Boson Peak of Vitreous Silica with the highfrequency Bulk Modulus derived from Brillouin Scattering Data, Phys. Rev. Lett. 104 (2010) 067402.

37. S. Sugai and A. Onodera Medium-range order in permanently densified $\mathrm{SiO}_{2}$ and $\mathrm{GeO}_{2}$ glass. Phys. Rev. Lett. 77 (1996) 4210-4213.

38. J. Schroeder, W. Wu, J. L. Apkarian, M. Lee, L. Hwa et al., Raman scattering and Boson peaks in glasses: temperature and pressure effects, J. Non-Cryst. Solids 349 (2004) 8897.

39. M. Zanatta, G. Baldi, S. Caponi, A. Fontana, E. Gilioli, M. Krish, C. Masciovecchio, G. Monaco, L. Orsingher, F. Rossi, G. Ruocco, and R. Verbeni Elastic properties of permanently densified silica: A Raman, Brillouin light, and x-ray scattering study.Phys. Rev. B 81 (2010) 212201.

40. R. J. Hemley, C Meade, and H.-K. Mao, Comment on "Medium-range order in permanently densified SiO2 and GeO2 glass", Phys. Rev. Lett. 79 (1997) 1420.

41. S. Reibstein, L. Wondraczek, D. de Ligny, S. Krolikowski, S. Sirotkin, J.-P. Simon, V. Martinez, B. Champagnon, Structural heterogeneity and pressure-relaxation in compressed borosilicate glasses by in situ small angle X-ray scattering, J. Chem. Phys. 134 (2011) 204502.

42. H. R. Schober, U. Buchenau, and V. L. Gurevich, Pressure dependence of the boson peak in glasses: Correlated and uncorrelated perturbations, Phys. Rev. B 89 (2014) 014204

43. T. Deschamps, C. Martinet, D. de Ligny, J. L. Bruneel, B. Champignon, Low-frequency Raman scattering under high pressure in diamond anvil cell: Experimental protocol and application to $\mathrm{GeO}_{2}$ and $\mathrm{SiO}_{2}$ boson peaks, J. Non-Cryst. Solids 358 (2012) 3156-3160.

44. A. I. Chumakov, G. Monaco, A. Fontana, A. Bosak, R. P. Hermann, D. Bessas, B. Wehinger, W. A. Crichton, M. Krisch, R. Rüffer, G. Baldi, G. Carini Jr., G. Carini, G. D'Angelo, E. Gilioli, G. Tripodo, M. Zanatta, B. Winkler, V. Milman, K. Refson, M. T. Dove, N. Dubrovinskaia, L. Dubrovinsky, R. Keding, and Y. Z. Yue, Role of Disorder in the Thermodynamics and Atomic Dynamics of Glasses, Phys. Rev. Lett. 112 (2014) 025502.

45. L. Wondraczek, Z. Pan, T. Palenta, A. Erlebach, S. T. Misture, M. Sierka, M. Micoulaut, U. Hoppe, J. Deubener, G. N. Greaves, Kinetics of decelerated melting, Adv. Sci. 5 (2018) 1700850.

46. P. F. McMillan, Structural studies of silicate-glasses and melts - applications and limitations of Raman-spectroscopy, Am. Min. 69 (1984) 622-644. 
47. S. K. Sharma, J. F. Mammone, M. F. Nicol, Raman investigation of ring configurations in vitreous silica, Nature 292 (1981) 140-141.

48. F. L. Galeener, Planar rings in glasses, Solid State Commun. 44 (1982) 1037.

49. P. Benassi, A. Fontana, W. Frizzera, M. Montagna, V. Mazzacurati, G. Signorelli, Disorder-induced light scattering in solids: The origin of the Boson peak in glasses, Philos. Mag. B 71 (1995) 761.

50. T. Rouxel, H. Ji, J. P. Guin, F. Augereau, B. Rufflé, Indentation deformation mechanism in glass: Densification versus shear flow, J. Appl. Phys. 107 (2010) 094903.

51. S. Yoshida, J.-C. Sanglebouf, T. Rouxel, Quantitative Evaluation of Indentation-Induced Densification in Glass, J. Mater. Res. 20 (2005) 3404-3412.

52. R. Shuker, R. W. Gammon, Raman-Scattering Selection-Rule Breaking and the Density of States in Amorphous Materials, Phys. Rev. Lett. 25 (1970) 222.

53. A. Fontana, R. Dell'Anna, M. Montagna, F. Rossi1, G. Viliani, G. Ruocco, M. Sampoli, U. Buchenau and A. Wischnewski, The Raman coupling function in amorphous silica and the nature of the long-wavelength excitations in disordered systems, Europhys. Lett., 47 (1999) 56.

54. N. V. Surovtsev and A. P. Sokolov, Frequency behavior of Raman coupling coefficient in glasses, Phys. Rev. B 66 (2002) 054205.

55. T.A. Michalske, D. Tallant, W.L. Smith, Raman Study of Silica Glass Under Tensile Stress, Phys. Chem. Glasses 29 (1988) 150-153.

56. Q. H. Le, T. Palenta, O. Benzine, K. Griebenow, R. Limbach, E. I. Kamitsos, L. Wondraczek, Formation, structure and properties of fluoro-sulfo-phosphate poly-anionic glasses, J. Non-Cryst. Solids 477 (2017) 58-72.

57. B. Champagnon, C. Martinet, M. Boudeulle, D. Vouagner, C. Coussa, T. Deschamps, L. Grosvalet. High pressure elastic and plastic deformations of silica: In situ diamond anvil cell Raman experiments, J. Non-Cryst. Solids 354 (2008) 569-573.

58. S. Susman, K. J. Volin, D. L. Price, M. Grimsditch, J. P. Rino, R. K. Kalia, P. Vashishta, G. Gwanmesia, Y. Wang, R. C. Liebermann, Intermediate-range order in permanently densified vitreous SiO2: A neutron-diffraction and molecular-dynamics study, Phys. Rev. B 43 (1991) 1194-1197

59. Devine RA, Arndt J. Si-O bond-length modification in pressure-densified amorphous SiO2. Phys. Rev. B, 35 (1987) 9376-9379.

60. R. L. Mozzi and B. E. Warren, The structure of vitreous silica, J. Appl. Crystallogr. 2 (1969) 164.

61. J. P. Rino, I. I. Ebbsjö, R. K. Kalia, A. Nakano, P. Vashishta, Structure of rings in vitreous SiO2, Phys. Rev. B 47 (1993) 3053.

62. K. Trachenko and M. T. Dove, Compressibility, kinetics, and phase transition in pressurized amorphous silica, Phys. Rev. B 67 (2003) 064107.

63. R. M. Van Ginhoven, H. Jónsson, L. R. Corrales, Silica glass structure generation for ab initio calculations using small samples of amorphous silica, Phys. Rev. B 71 (2005) 024208.

64. Y. Inamuraa, M. Arai, N. Kitamura, S.M. Bennington, A.C. Hannon, Intermediate-range structure and low-energy dynamics of densified $\mathrm{SiO}_{2}$ glass, Physica B 241-243 (1998) 903. 
65. U. Buchenau, M. Prager, N. Nücker, A. J. Dianoux, N. Ahmad, and W. A. Phillips Lowfrequency modes in vitreous silica. Phys. Rev. B 34 (1986) 5665.

66. Fabiani E, Fontana A, Buchenau U. Neutron scattering study of the vibrations in vitreous silica and germania. J. Chem. Phys. 128 (2008) 244507.

67. C. Weigel, M. Foret, B. Hehlen, M. Kint, S. Clément, A. Polian, R. Vacher, and B. Rufflé, Polarized Raman spectroscopy of $\mathrm{V}^{-\mathrm{SiO}_{2}}$ under rare-gas compression. Phys. Rev. B 93 (2016) 224303.

68. H. M. Flores-Ruiz, G. G. Naumis, The transverse nature of the Boson peak: A rigidity theory approach, Physica B 418 (2013) 26-31.

69. S. Sawamura, R. Limbach, H. Behrens, L. Wondraczek, Lateral deformation and defect resistance of compacted silica glass: quantification of the scratching hardness of brittle glasses, J. Non-Cryst. Solids 481 (2018) 503-511.

70. T. Deschamps, J. Margueritat, C. Martinet, A. Mermet, B. Champagnon. Elastic moduli of permanently densified silica glasses, Sci. Rep. 4 (2014) 7193.

71. K. Niss, B. Begen, B. Frick, J. Ollivier, A. Beraud, A. Sokolov, V. N. Novikov, C. AlbaSimionesco, Influence of Pressure on the Boson Peak: Stronger than Elastic Medium Transformation, Phys. Rev. Lett. 99 (2007) 055502.

72. B. Mantisi, S. Adichtchev, S. Sirotkin, L. Rafaelly, L. Wondraczek, H. Behrens, C. Marcenat, N. V. Surovtsev, A. Pillonnet, E. Duval, Non-Debye normalization of the glass vibrational density of states in mildly densified silicate glasses, J. Phys.: Condens. Matter 22 (2010) 025402, 1-5.

73. F. Leonforte, Vibrational states and disorder in continuously compressed model glasses, J. Non-Cryst. Solids 357 (2011) 552-558.

74. S.N. Yannopoulosa, K.S. Andrikopoulos, G. Ruocco, On the analysis of the vibrational Boson peak and low-energy excitations in glasses, J. Non-Cryst. Solids 352 (2006) 45414551.

75. T. Deschamps, C. Martinet, D.R. Neuville, D.de Ligny, C. Coussa-Simon, B. Champagnon, Silica under hydrostatic pressure: A non-continuous medium behaviour, $\mathrm{J}$. Non-Cryst. Solids 355 (2009) 2422-2424.

76. E. Duval, A. Mermet, and L. Saviot, Boson peak and hybridization of acoustic modes with vibrations of nanometric heterogeneities in glasses, Phys. Rev. B 75 (2007) 024201.

77. E. Duval, A. Boukenter, B. Champagnon, Vibration Eigenmodes and Size of Microcrystallites in Glass: Observation by Very-Low-Frequency Raman Scattering, Phys. Rev. Lett. 56 (1986) 2052.

78. U. Tracht, M. Wilhelm, A. Heuer, H. Feng, K. Schmidt-Rohr, and H. W. Spiess, Length Scale of Dynamic Heterogeneities at the Glass Transition Determined by Multidimensional Nuclear Magnetic Resonance, Phys. Rev. Lett. 81 (1998) 2727-2730.

79. C. Hühn, A. Erlebach, D. Mey, L. Wondraczek, M. Sierka, Ab initio energetics of Si-O bond cleavage, J. Comput. Chem. 38 (2017) 2349-2353.

80. F. Léonforte, A. Tanguy, J. P. Wittmer, J. L. Barrat, Inhomogeneous Elastic Response of Silica Glass, Phys. Rev. Lett. 97 (2006) 055501.

81. A. Sokolov, A. Kisliuk, M. Soltwisch, D. Quitmann, Medium range order in glasses: Comparison of Raman and diffraction measurements, Phys. Rev. Lett. 69 (1992) 1540.

82. S. R. Elliott, A Unified Model for the Low-Energy Vibrational Behaviour of Amorphous Solids, Europhys. Lett. 19 (1992) 201-206. 
83. S. R. Elliott, Medium-range structural order in covalent amorphous solids, Nature 354 (1991) 445-452.

84. L. Börjesson, A. K. Hassan, J. Swenson, L. M. Torell, A. Fontana, Is there a correlation between the first sharp diffraction peak and the low frequency vibrational behavior of glasses?, Phys. Rev. Lett. 70 (1993) 1275.

85. Dassault Systèmes, 10 rue Marcel Dassault, 78140 Vélizy-Villacoublay, France

86. R. Limbach, B. P. Rodrigues, L. Wondraczek, Strain-rate sensitivity of glasses, J. NonCryst. Solids 404 (2014) 124-134.

87. T. Rouxel, H. Ji, T. Hammouda, A. Moréac, Poisson's Ratio and the Densification of Glass under High Pressure, Phys. Rev. Lett. 100 (2008) 225501 (2008).

\section{Acknowledgments}

This project has received funding from the European Research Council (ERC) under the European Union's Horizon 2020 research and innovation program (ERC grant UTOPES, grant agreement no. 681652). Further financial support through the priority program PP 1594 of the German Science Foundation is gratefully acknowledged. The authors would like to thank Thierry Deschamps for providing BLS data.

\section{Author Contributions Statement}

L.W. initiated and supervised the project. O.M. conducted indentation experiments and low-frequency Raman analyses. O. B., Z. P. and L.W. evaluated Boson peak data and performed VDoS scalings. S.B. and K.D. performed FEM simulations. O.B. and L.W. wrote the manuscript with input from all authors. All authors were involved in manuscript revisions.

\section{Competing Financial Interests Statement}

We declare that the authors have no competing financial interests, or other interests that might be perceived to influence the results and discussion reported in this paper.

\section{Data Availability Statement}

The datasets generated during and/or analysed during the current study are available from the corresponding author on reasonable request. 